\title{
Effect of Ambient Temperature on Total Organic Gas Speciation Profiles from Light- Duty Gasoline Vehicle Exhaust
}

\section{Supporting Information}

\author{
Anirban Roy ${ }^{1}$, Darrell Sonntag ${ }^{2}$, Richard Cook ${ }^{2}$, Catherine Yanca ${ }^{2}$, Charles Schenk ${ }^{2}$, and \\ Yunsoo $\mathrm{Choi}^{{ }^{*}}$ \\ ${ }^{1}$ Department of Earth and Atmospheric Sciences, University of Houston, Houston, Texas 77004. \\ ${ }^{2}$ United States Environmental Protection Agency’s Office of Transportation and Air Quality, \\ Ann Arbor, Michigan 48105.
}

This document contains five (5) figures and eight (8) tables.

Figure S1: Traces of the drive cycles considered in this study.

Figure S2: Comparison of speciation profiles for units of $\mathrm{mg} \mathrm{mi}^{-1}$ and $\mathrm{mg} \mathrm{kg-fuel}^{-1}$ for the FTP75-composite emissions at $0^{\circ} \mathrm{F}$.

Figure S3: Comparison of Total Hydrocarbon emission factors from this study vis-à-vis those in the literature.

Figure S4: Comparison of NonMethane Hydrocarbon emission factors using two different methods to quantify unspeciated compounds.

Figure S5: Box plot of NMOG-NMHC ratios for the vehicle fleet tested. Star represents the fleetaverage.

Table S1: Fuel Speciation.

Table S2: Fuel characteristics nationwide

Table S3: List of oxygenated species measured in exhaust

Table S4a: Mean emission factors and corresponding uncertainty, FTP75 cycle; units mg mi ${ }^{-1}$.

Table S4b: Mean emission factors and corresponding uncertainty, US06 cycle; units in mg mi ${ }^{-1}$.

Table S5a: Emission factors for the US06 drive cycle and Cold Start phase in units of mg kgfuel $^{-1}$.

Table S5b: Emission factors for the FTP75-Composite phase in units of mg kg-fuel ${ }^{-1}$.

Table S6: Ratios of mean emission factors in the running and hot start stages to cold start.

Table S7: Uncertainty in US06 vis-à-vis running emissions at $75^{\circ} \mathrm{F}$.

Table S8: Probability of speciation fractions being equal across all temperatures for the US06 cycle. 

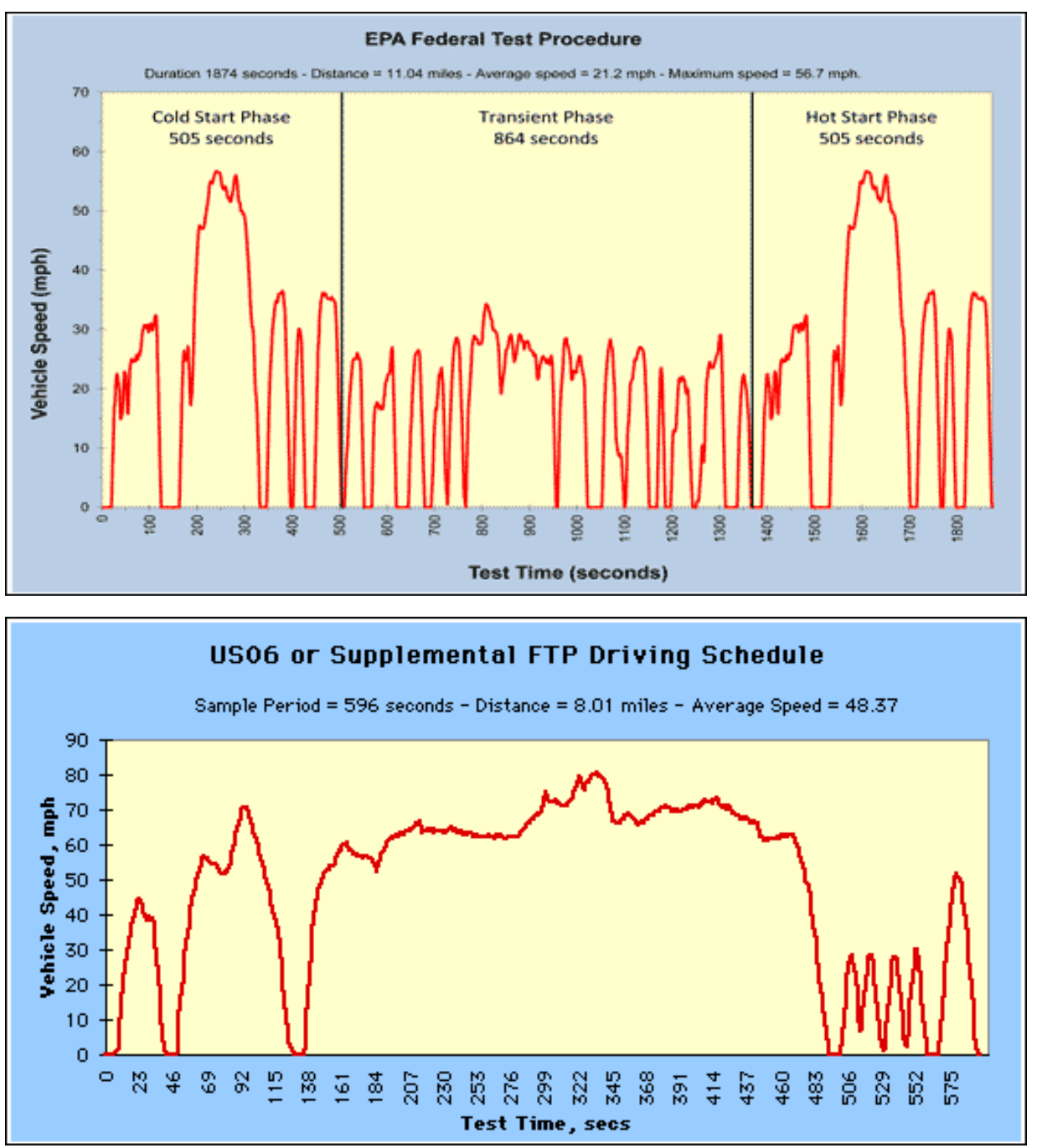

Figure S1: Traces of the drive cycles considered in this study.

\section{Fuel Characteristics}

\section{Table S1: Fuel Speciation.}

\begin{tabular}{|c|c|c|}
\hline SPECIES & Weight \% & Species Class \\
\hline Ethanol & 9.78 & Alcohols, $\sim 10 \%$ \\
\hline Propane & 0.05 & \multirow{7}{*}{ Alkanes, 41\% } \\
\hline Isobutane & 0.17 & \\
\hline N-Butane & 2.98 & \\
\hline 2,2-Dimethylpropane & 0.14 & \\
\hline Isopentane & 5.95 & \\
\hline N-Pentane & 3.9 & \\
\hline 2,2-Dimethylbutane & 0.55 & \\
\hline
\end{tabular}




\begin{tabular}{|c|c|c|}
\hline 2,3-Dimethylbutane & 1.06 & \\
\hline 2-Methylpentane & 2.94 & \\
\hline 3-Methylpentane & 2.45 & \\
\hline N-Hexane & 6.07 & \\
\hline 2,2-Dimethylpentane & 0.39 & \\
\hline 2,4-Dimethylpentane & 0.57 & \\
\hline 2,2,3-Trimethylbutane & 0.12 & \\
\hline 3,3-Dimethylpentane & 0.16 & \\
\hline 2-Methylhexane & 1.3 & \\
\hline 2,3-Dimethylpentane & 0.43 & \\
\hline 3-Methylhexane & 1.03 & \\
\hline 3-Ethylpentane & 0.05 & \\
\hline 2,2,4-Trimethylpentane & 1.29 & \\
\hline N-Heptane & 1.33 & \\
\hline 2,4,4-Trimethyl-1-pentene & 1.53 & \\
\hline 2,2-Dimethylhexane & 0.11 & \\
\hline 2,5-Dimethylhexane & 0.33 & \\
\hline 2,4-Dimethylhexane & 0.25 & \\
\hline 3,3-Dimethylhexane & 0.04 & \\
\hline 2,3,4-Trimethylpentane & 0.53 & \\
\hline 2,3,3-Trimethylpentane & 0.49 & \\
\hline 2,3-Dimethylhexane & 0.21 & \\
\hline 2-Methylheptane & 0.4 & \\
\hline 4-Methylheptane & 0.17 & \\
\hline 3-Methylheptane & 0.31 & \\
\hline N-Octane & 0.7 & \\
\hline 2,5-Dimethylheptane & 0.11 & \\
\hline 3,5-Dimethylheptane & 0.03 & \\
\hline 2,3-Dimethylheptane & 0.04 & \\
\hline 3,4-Dimethylheptane D/L & 0.02 & \\
\hline 4-Ethylheptane & 0.01 & \\
\hline 4-Methyloctane & 0.08 & \\
\hline 2-Methyloctane & 0.11 & \\
\hline 3-Ethylheptane & 0.02 & \\
\hline 3-Methyloctane & 0.1 & \\
\hline 2,2,6-Trimethylheptane & 0.01 & \\
\hline N-Nonane & 0.29 & \\
\hline 2,4-Dimethyloctane & 0.05 & \\
\hline 2,6-Dimethyloctane & 0.08 & \\
\hline 2,5-Dimethyloctane & 0.02 & \\
\hline 3,5-Dimethyloctane & 0.12 & \\
\hline
\end{tabular}




\begin{tabular}{|c|c|c|}
\hline 2,3-Dimethyloctane & 0.01 & \\
\hline 3-methyl-5-ethylheptane & 0.04 & \\
\hline 4-Ethyloctane & 0.02 & \\
\hline 4-Methylnonane & 1.06 & \\
\hline 2-Methylnonane & 0.04 & \\
\hline 3-Ethyloctane & 0.01 & \\
\hline 3-Methylnonane & 0.05 & \\
\hline N-Decane & 0.1 & \\
\hline N-Tridecane & 0.01 & \\
\hline 2,2,5-Trimethylhexane & 0.03 & \\
\hline 2,4-Dimethylheptane & 0.05 & \\
\hline 2,6-Dimethylheptane & 0.09 & \\
\hline N-Undecane & 0.13 & \\
\hline Methylcyclopentane & 1.89 & \multirow[t]{28}{*}{ Cyclic Alkanes, $~ 11 \%$} \\
\hline Cyclohexane & 2.87 & \\
\hline 1,1-Dimethylcyclopentane & 0.16 & \\
\hline Cis-1,3-dimethylcyclopentane & 0.19 & \\
\hline Trans-1,3-dimethylcyclopentane & 0.17 & \\
\hline Trans-1,2-dimethylcyclopentane & 0.27 & \\
\hline Methylcyclohexane & 1.91 & \\
\hline Ethylcyclopentane & 0.07 & \\
\hline Trans,cis-1,2,4-trimethylcyclopentane & 0.05 & \\
\hline Trans,cis-1,2,3-trimethylcyclopentane & 0.05 & \\
\hline Cis-1,3 dimethylcyclohexane & 0.32 & \\
\hline Trans-1,4-dimethylcyclohexane & 0.16 & \\
\hline 1,1-Dimethylcyclohexane & 0.07 & \\
\hline Trans-1-ethyl-3-methylcyclopentane & 0.23 & \\
\hline Cis-1-ethyl-3-methylcyclopentane & 0.02 & \\
\hline Trans-1-ethyl-2-methylcyclopentane & 0.02 & \\
\hline Trans-1,2,dimethylcyclohexane & 0.12 & \\
\hline Trans-1,3,dimethylcyclohexane & 0.06 & \\
\hline Cis-1,4-dimethylcyclohexane & 0.06 & \\
\hline Isopropylcyclopentane & 0.01 & \\
\hline Cis-1-ethyl-2-methylcyclopentane & 0.04 & \\
\hline Cis-1,2-dimethylcyclohexane & 0.02 & \\
\hline Ethylcyclohexane & 0.15 & \\
\hline 1,1,3-Trimethylcyclohexane & 0.05 & \\
\hline C9 Naphthene & 0.01 & \\
\hline Cis,trans,1,3,5-trimethylcyclohexane & 0.04 & \\
\hline Cis,cis,1,2,4,trimethylcyclohexane & 0.01 & \\
\hline Trans-1-Methyl-2-propylcyclopentane & 0.02 & \\
\hline
\end{tabular}




\begin{tabular}{|c|c|c|}
\hline Cis-1-ethyl-3-methylcyclohexane & 0.05 & \\
\hline Trans-1-ethyl-4-methylcyclohexane & 0.02 & \\
\hline Isobutylcyclopentane & 0.02 & \\
\hline Trans-1-ethyl-3-methylcyclohexane & 0.01 & \\
\hline Trans-1-ethyl-2-methylcyclohexane & 0.02 & \\
\hline Cis-1-ethyl-4-methylcyclohexane & 0.02 & \\
\hline Sec-butylcyclopentane & 0.08 & \\
\hline N-propylcyclohexane & 0.05 & \\
\hline N-butylcyclopentane & 0.04 & \\
\hline C10 Naphthene & 0.05 & \\
\hline 1,2,3,4 Tet-methylcyclohexane & 0.46 & \\
\hline C10 Naphthenes & 0.12 & \\
\hline Cis-1-methyl-3-propylcyclohexane & 0.19 & \\
\hline Cis 1,3 diethylcyclohexane & 0.02 & \\
\hline Trans-1-methyl-3-propylcyclohexane & 0.3 & \\
\hline Trans 1,3 diethylcyclohexane & 0.07 & \\
\hline Butylcyclohexane & 0.05 & \\
\hline C11 Naphthenes & 0.03 & \\
\hline 1-Hexene & 2.41 & \multirow{2}{*}{ Alkenes, 3\% } \\
\hline 2,4,4-Trimethyl-1-pentene & 0.41 & \\
\hline Benzene & 0.71 & \multirow{21}{*}{ Aromatics, 35\% } \\
\hline Toluene & 11.21 & \\
\hline Ethylbenzene & 1.28 & \\
\hline Meta-Xylene & 3.26 & \\
\hline Para-Xylene & 1.4 & \\
\hline Ortho-Xylene & 1.64 & \\
\hline Isopropylbenzene & 0.18 & \\
\hline n-Propylbenzene & 0.64 & \\
\hline 1-Methyl-3-ethylbenzene & 2.26 & \\
\hline 1-Methyl-4-ethylbenzene & 1.04 & \\
\hline 1,3,5-Trimethylbenzene & 1.34 & \\
\hline 1-Methyl-2-ethylbenzene & 1.5 & \\
\hline 1,2,4-Trimethylbenzene & 4.52 & \\
\hline Isobutylbenzene & 0.31 & \\
\hline sec-Butylbenzene & 0.24 & \\
\hline 1,2,3-Trimethylbenzene & 0.86 & \\
\hline 1-Methyl-3-isopropylbenzene & 0.14 & \\
\hline 1-Methyl-4-isopropylbenzene & 0.04 & \\
\hline Indan (2,3-Dihydroindene) & 0.02 & \\
\hline 1-Methyl-2-isopropylbenzene & 0.3 & \\
\hline 1,3-Diethylbenzene & 0.02 & \\
\hline
\end{tabular}




\begin{tabular}{|l|l|}
\hline 1-Methyl-3-n-propylbenzene & 0.05 \\
\hline 1,4-Diethylbenzene & 0.01 \\
\hline N-Butylbenzene & 0.01 \\
\hline 1,3-Dimethyl-5-ethylbenzene & 0.48 \\
\hline 1-Methyl-2-n-propylbenzene & 0.2 \\
\hline 1,4-Dimethyl-2-ethylbenzene & 0.21 \\
\hline 1,3-Dimethyl-4-ethylbenzene & 0.31 \\
\hline 1,2-Dimethyl-4-ethylbenzene & 0.08 \\
\hline 1,3-Dimethyl-2-ethylbenzene & 0.12 \\
\hline 1,2-Dimethyl-3-Ethylbenzene & 0.07 \\
\hline 1,2,4,5-Tetramethylbenzene & 0.01 \\
\hline 1,2,3,5-Tetramethylbenzene & 0.03 \\
\hline 4-Methylindan & 0.01 \\
\hline 5-Methylindan & 0.03 \\
\hline 1,2,3,4-Tetramethylbenzene & 0.01 \\
\hline Pentylbenzene & 0.01 \\
\hline C11 Aromatic & 0.59 \\
\hline 2-Methylnaphthalene & 0.01 \\
\hline C12 Unidentified & 0.01 \\
\hline Unidentified & 0.87 \\
\hline
\end{tabular}

Table S2: Fuel characteristics nationwide

\begin{tabular}{|l|l|l|l|l|c|c|c|c|}
\hline \multirow{2}{*}{ Property } & & \multicolumn{2}{c|}{ Test Fuel } & \multicolumn{2}{c|}{$\begin{array}{c}\text { 2009 AAM Summer } \\
\text { Fuel Survey }\end{array}$} & \multicolumn{2}{c|}{$\begin{array}{c}\text { 2009 AAM Winter } \\
\text { Fuel Survey }\end{array}$} \\
\cline { 2 - 11 } & Test Method & Units & \multicolumn{1}{|c|}{ S } & W & Mean & Range & Mean & Range \\
\hline Ethanol & ASTM D-5599 & Vol\% & 9.9 & 10.2 & 9.4 & $5.4-10.6$ & 9.4 & $5.4-10.7$ \\
\hline Sulfur & ASTM D-2622 & ppm & 7 & 8 & 30.9 & $2-238$ & 35.9 & $6-220$ \\
\hline & & & & & & & & \\
Vapor pressure & ASTM D-5191 & psi & 9.3 & 11.4 & 8.1 & $6.8-10$ & 12.8 & $8.6-15.5$ \\
\hline Benzene & ASTM D-3606 & Vol\% & 0.61 & 0.58 & 0.9 & $0.3-23$ & 0.9 & $0.4-2$ \\
\hline Aromatics & ASTM D-5769 & Vol\% & 24.8 & 23.5 & 23.2 & $9.7-34$ & 20.1 & $12.3-28.2$ \\
\hline Olefins & ASTM D-1319 & Vol\% & 4 & 3.8 & 7.2 & $0.6-15.7$ & 8 & $2-18$ \\
\hline T50 & ASTM D-86 & ${ }^{0} \mathrm{~F}$ & 180 & 161 & 197 & $153-222$ & 161 & $141-210$ \\
\hline T90 & ASTM D-86 & ${ }^{\circ} \mathrm{F}$ & 312 & 311 & 331 & $285-356$ & 308 & $268-343$ \\
\hline & & & & & & & & \\
Specific Gravity & ASTM D-4052 & & 0.75 & 0.74 & 0.747 & $0.73-0.76$ & 0.729 & $0.71-0.746$ \\
\hline
\end{tabular}

Table S3: List of oxygenated species measured in exhaust

\begin{tabular}{|l|l|}
\hline Oxygenate Compound & Species Class \\
\hline Acetaldehyde & \\
\cline { 1 - 1 } Acrolein & \\
\cline { 1 - 1 } Benzaldehyde & \\
\cline { 1 - 1 } Crotonaldehyde & \multirow{3}{*}{ Aldehydes } \\
\cline { 1 - 1 } Formaldehyde & \\
\cline { 1 - 1 } Hexanal & \\
\cline { 1 - 1 } Methacrolein & \\
\cline { 1 - 1 } Propionaldehyde & \\
\hline
\end{tabular}




\begin{tabular}{|l|l|}
\hline$n$-Butyraldehyde & \\
\cline { 1 - 1 }$n$-Valeraldehyde & \\
\cline { 1 - 1 } p-Tolylaldehyde & \multirow{2}{*}{ Ketones } \\
\cline { 1 - 1 } Acetone & \\
\hline Methylethylketone & Alcohols \\
\cline { 1 - 1 } Methanol & \\
\cline { 1 - 2 } Ethanol & Ethers \\
\hline m-tertiary butyl ether &
\end{tabular}

Table S4a: Mean emission factors and corresponding uncertainty, FTP75 cycle; units in mg mi ${ }^{-}$ 1 .

\begin{tabular}{|l|c|c|c|c|c|c|}
\hline \multirow{2}{*}{ Species } & \multicolumn{3}{|c|}{ Cold Start } & \multicolumn{3}{c|}{ Composite } \\
\cline { 2 - 7 } & $\mathbf{7 5}{ }^{\mathbf{0}} \mathbf{F}$ & $\mathbf{2 0}{ }^{\mathbf{}} \mathbf{F}$ & $\mathbf{0}^{\mathbf{0}} \mathbf{F}$ & $\mathbf{7 5}^{\mathbf{0}} \mathbf{F}$ & $\mathbf{2 0}{ }^{\mathbf{}} \mathbf{F}$ & $\mathbf{0}^{\mathbf{}} \mathbf{F}$ \\
\hline Aromatics & $54(39-71)$ & $388(285-499)$ & $1268(819-1758)$ & $12(9-15)$ & $83(61-106)$ & $269(176-371)$ \\
\hline Alkanes & $33(28-39)$ & $302(245-351)$ & $1259(921-1593)$ & $8(7-10)$ & $66(54-76)$ & $266(195-336)$ \\
\hline $\begin{array}{l}\text { Cyclic } \\
\text { Alkanes }\end{array}$ & $5(3-6)$ & $54(36-72)$ & $192(104-290)$ & $1(0.8-1.3)$ & $11(8-15)$ & $40(22-60)$ \\
\hline Alkenes & $23(17-30)$ & $191(149-232)$ & $622(477-760)$ & $5(3-6)$ & $40(31-48)$ & $129(99-158)$ \\
\hline Alkynes & $2(1.1-2.9)$ & $17(13-22)$ & $90(61-118)$ & $0.4(0.2-0.6)$ & $4(3-5)$ & $19(13-25)$ \\
\hline Alcohols & $8.4(4.1-15)$ & $53(42-67)$ & $244(167-323)$ & $7.3(2.7-13.5)$ & $15(10-23)$ & $58(41-75)$ \\
\hline Aldehydes & $7(5-11)$ & $18(15-21)$ & $33(27-41)$ & $3(2.4-4.0)$ & $7(6-9)$ & $8.8(7.5-10.4)$ \\
\hline Ketones & $1(0.8-1.3)$ & $3.2(1.7-5.8)$ & $3(2.5-3.7)$ & $0.6(0.4-0.7)$ & $1.9(1.1-2.9)$ & $1.1(0.95-1.3)$ \\
\hline Methane & $12(9-15)$ & $46(37-55)$ & $140(114-167)$ & $5(4-7)$ & $13(11-15)$ & $35(29-40)$ \\
\hline
\end{tabular}

Table S4b: Mean emission factors and corresponding uncertainty, US06 cycle; units in mg mi ${ }^{-1}$.

\begin{tabular}{|l|c|c|c|}
\hline Species & $\mathbf{7 5}^{\mathbf{}} \mathbf{F}$ & $\mathbf{2 0}{ }^{\mathbf{0}} \mathbf{F}$ & $\mathbf{0}^{\mathbf{0}} \mathbf{F}$ \\
\hline Aromatics & $3(2-6)$ & $7(4-10)$ & $22(15-29)$ \\
\hline Alkanes & $3.6(2.5-4.8)$ & $6(3-8)$ & $9(6-11)$ \\
\hline $\begin{array}{l}\text { Cyclic } \\
\text { Alkanes }\end{array}$ & $0.25(0.08-0.4)$ & $0.23(0.05-0.44)$ & $0.35(0.13-0.63)$ \\
\hline Alkenes & $2.2(0.97-3.56)$ & $3.1(1.4-4.9)$ & $4(2-5)$ \\
\hline Alkynes & $0.08(0-0.19)$ & $0.13(0.005-0.3)$ & $0.11(0.04-0.19)$ \\
\hline Alcohols & $2.1(0.7-3.7)$ & $1.1(0.3-2.2)$ & $1.4(0.5-2.4)$ \\
\hline Aldehydes & $0.76(0.3-1.5)$ & $3(1-6)$ & $1.2(0.7-1.9)$ \\
\hline Ketones & $0.16(0.07-0.25)$ & $0.16(0.1-0.23)$ & $0.6(0.09-1.6)$ \\
\hline Methane & $5.1(3.5-6.5)$ & $5(3-7)$ & $7(5-10)$ \\
\hline
\end{tabular}

\section{Emission factors on a unit fuel basis}

We calculated fuel-based emission factors for each species classes, the results for these calculations are listed in Tables S4a and S4b. The calculation is done by the following equation:

$$
E F\left(m g k g \mathrm{fuel}^{-1}\right)=\frac{E F\left(m g m i^{-1}\right) \times E \times C}{\rho_{\text {gasoline }}}
$$

Where $\mathrm{E}$ is the fuel economy distribution in miles per gallon, $\mathrm{C}$ is the conversion factor (1 gallon $=3.8$ litres), and $\rho_{\text {gasoline }}$ is the density of gasoline, 0.75 grams litre ${ }^{-1}$. This equation could be 
used for stand-alone emissions such as Cold Start and the US06, but the relation between distance and fuel-based emission factors is non-linear, which could cause difference in speciation profiles. However, our results indicated that there was negligible change in the speciation profiles by using fuel-based EFs vis-à-vis distance-based EFs.

Table S5a: Emission factors for the US06 drive cycle and cold start phase, units mg kg-fuel ${ }^{-1}$.

\begin{tabular}{|c|c|c|c|c|c|c|}
\hline & \multicolumn{3}{|c|}{ US06 } & \multicolumn{3}{|c|}{ FTP-75 Cold Start $/ \mathbf{1 0}^{3}$} \\
\hline & $\mathrm{O}^{0} \mathrm{~F}$ & $20^{\circ} \mathrm{F}$ & $75^{\circ} \mathrm{F}$ & $0^{0} \mathrm{~F}$ & $20^{\circ} \mathrm{F}$ & $75^{\circ} \mathrm{F}$ \\
\hline Aromatics & $180(125-234)$ & $55(35-78)$ & 34 (19-50) & $7(5-10)$ & $2.5(1.8-3.1)$ & $0.4(0.3-0.6)$ \\
\hline Alkanes & $69(49-91)$ & $46(28-64)$ & $31(21-41)$ & $7(5-9)$ & $1.9(1.5-2.3)$ & $0.27(0.2-0.32)$ \\
\hline Cyclic Alkanes & $2.9(1.1-5.2)$ & $11.8(0.4-3.5)$ & $2.2(0.7-3.7)$ & $1(0.6-1.7)$ & $0.35(0.24-0.46)$ & $0.04(0.03-0.05)$ \\
\hline Alkenes & 31 (19-44) & $26(12-41)$ & $19(9-30)$ & $3.6(2.7-4.4)$ & $1.2(0.9-1.5)$ & $0.19(0.13-0.25)$ \\
\hline Alkynes & $0.9(0.4-1.6)$ & $1.1(0.06-2.4)$ & $0.65(0-1.5)$ & $0.5(0.3-0.7)$ & $0.11(0.08-0.14)$ & $\mathrm{n} / \mathrm{a}$ \\
\hline Alcohols & $11(4-20)$ & $9(2-17)$ & $18(7-31)$ & $1.4(1-2)$ & $0.34(0.26-0.42)$ & $0.07(0.03-0.12)$ \\
\hline Aldehydes & $10(5-14)$ & $23(9-47)$ & $6.5(2.5-12.5)$ & $0.19(0.15-0.24)$ & $0.1(0.09-0.13)$ & $0.06(0.04-0.09)$ \\
\hline Ketones & $5(1-14)$ & $1.3(0.8-1.9)$ & $1.3(0.6-2.1)$ & $\mathrm{n} / \mathrm{a}$ & $0.02(0.01-0.04)$ & $\mathrm{n} / \mathrm{a}$ \\
\hline Methane & 59 (41-78) & $41(25-58)$ & $43(29-57)$ & $0.8(0.6-1)$ & $0.3(0.02-0.35)$ & $0.1(0.07-0.13)$ \\
\hline
\end{tabular}

Table S5b: Emission factors for the FTP75-Composite phase, units mg kg-fuel ${ }^{-1}$.

\begin{tabular}{|l|c|c|c|}
\hline \multirow{2}{*}{ Aromatics } & \multicolumn{3}{|c|}{ FTP 75Composite Emission Factors $\mathbf{( m g ~ k g ~ f u e l ~}^{-1}$ ) } \\
\cline { 2 - 4 } & $1800(1200-2500)$ & $600(400-700)$ & $100(70-120)$ \\
\hline Alkanes & $1800(1200-2300)$ & $450(370-530)$ & $70(50-80)$ \\
\hline Cyclic Alkanes & $270(140-410)$ & $70(50-100)$ & $9(7-11)$ \\
\hline Alkenes & $880(680-1100)$ & $270(210-330)$ & $40(30-50)$ \\
\hline Alkynes & $130(90-170)$ & $20(10-30)$ & $3(2-5)$ \\
\hline Alcohols & $400(300-500)$ & $100(70-160)$ & $60(20-120)$ \\
\hline Aldehydes & $60(50-70)$ & $50(40-60)$ & $26(20-34)$ \\
\hline Ketones & $7.8(6.7-9.1)$ & $10(8-20)$ & $5(4-6)$ \\
\hline Methane & $242(198-283)$ & $90(70-100)$ & $40(30-60)$ \\
\hline
\end{tabular}

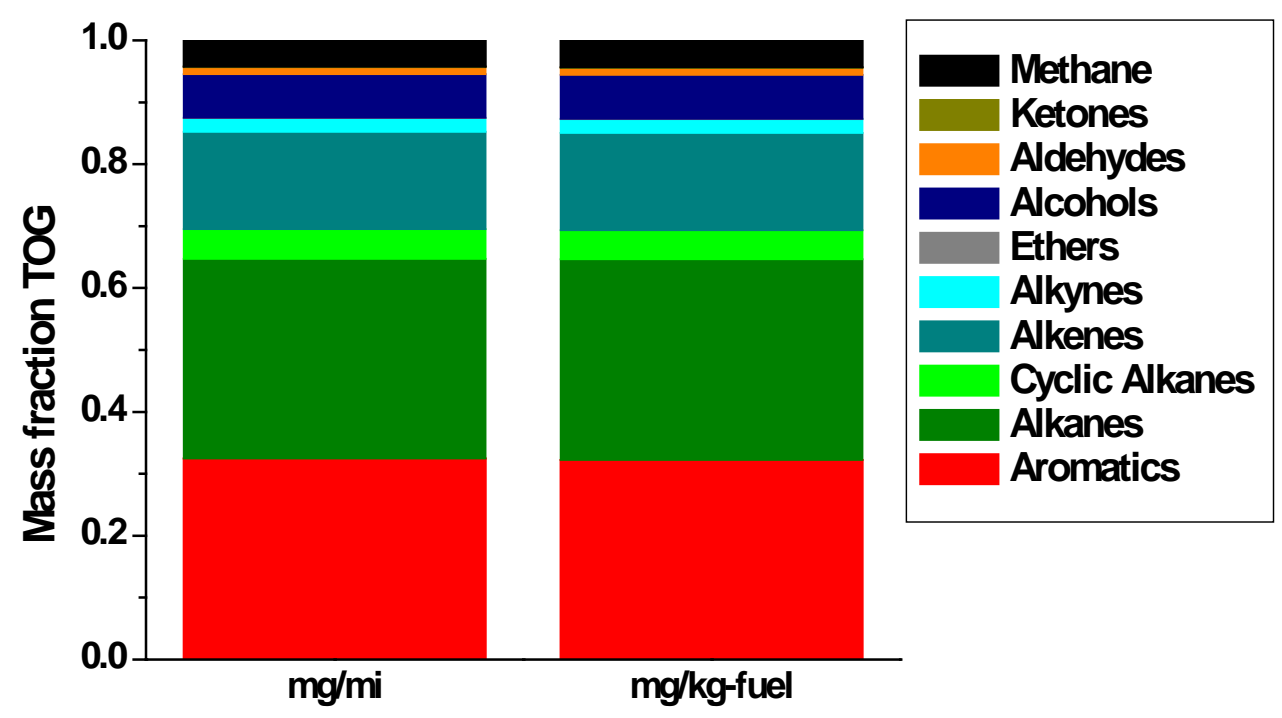

Figure S2: Comparison of speciation profiles for units of mg mi ${ }^{-1}$ and $\mathrm{mg} \mathrm{kg}^{-f u e l}{ }^{-1}$ for the FTP75-composite emissions at $0^{\circ} \mathrm{F}$. 


\section{Emissions of new vs. old vehicles}

The data reported are raw values representing test-to-test and inter-unit variability. For each temperature in our study, the emission factors represent FTP75-composites and US06 aggregates. For each of the cases, the star symbol represents the mean of the distribution. The source of literature data are Stump et al. (2005a, b, c) and Stanard et al. (2005). Some of the vehicles were purposefully tested for faulty conditions; for example the oxygen sensor disconnected or the exhaust gas recirculator turned off.

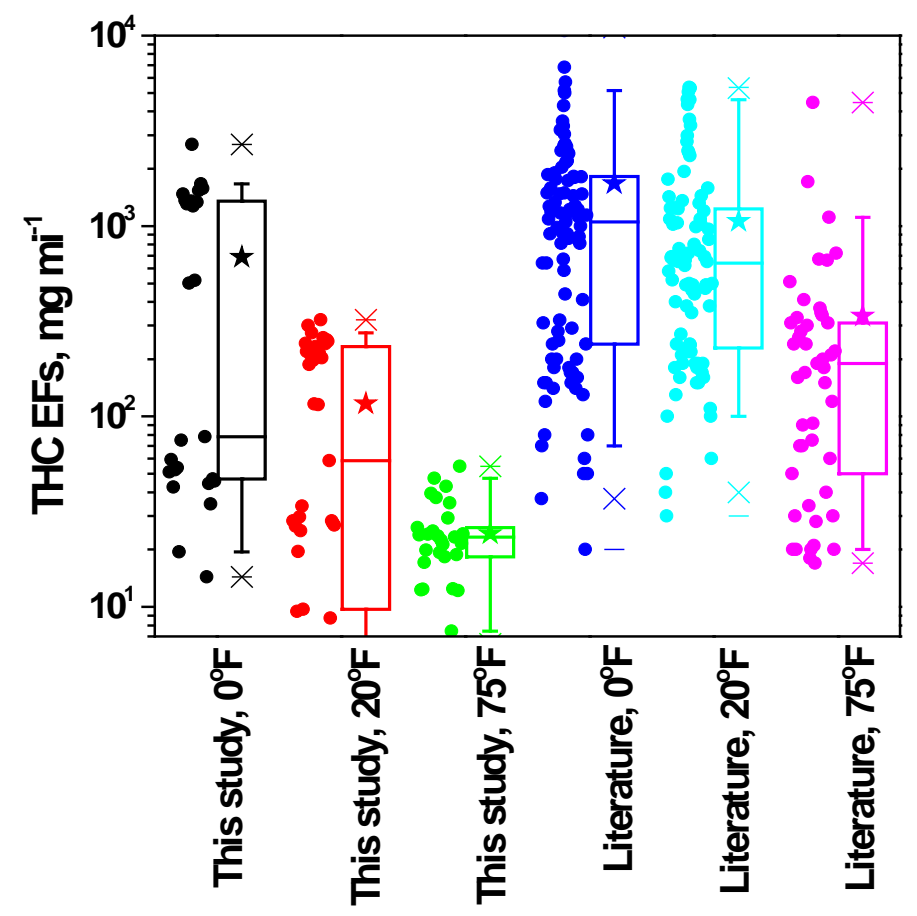

Figure S3: Comparison of Total Hydrocarbon emission factors from this study vis-à-vis those in the literature. 


\section{Comparison of phase-specific emissions for the FTP75 cycle}

Table S6: Ratios of mean emission factors in the running and hot start stages to cold start.

\begin{tabular}{|c|c|c|c|c|c|c|}
\hline \multirow{3}{*}{ Species } & \multicolumn{6}{|c|}{ Ratios of emissions to cold start } \\
\hline & \multicolumn{3}{|c|}{ Running } & \multicolumn{3}{|c|}{ Hot Start } \\
\hline & $\mathbf{0}^{0} \mathrm{~F}$ & $20^{\circ} \mathrm{F}$ & $75^{\circ} \mathrm{F}$ & $\mathbf{0}^{\circ} \mathbf{F}$ & $20^{\circ} \mathrm{F}$ & $75^{\circ} \mathrm{F}$ \\
\hline Aromatics & $\begin{array}{c}8.5 \times 10^{-3} \\
\left(4.6 \times 10^{-3}-0.015\right)\end{array}$ & $\begin{array}{c}7.3 \times 10^{-3} \\
\left(4 \times 10^{-3}-1.2 \times 10^{-2}\right)\end{array}$ & $\begin{array}{c}1.3 \times 10^{-2} \\
\left(7.3 \times 10^{-3}-2.2 \times 10^{-2}\right)\end{array}$ & $\begin{array}{c}2.9 \times 10^{-3} \\
\left(1.7 \times 10^{-3}-4.8 \times 10^{-3}\right)\end{array}$ & $\begin{array}{c}9.4 \times 10^{-3} \\
\left(5.9 \times 10^{-3}-1.4 \times 10^{-2}\right)\end{array}$ & $\begin{array}{c}2.4 \times 10^{-2} \\
\left(1.2 \times 10^{-2}-3.2 \times 10^{-2}\right)\end{array}$ \\
\hline Alkanes & $\begin{array}{c}4.5 \times 10^{-3} \\
\left(2.2 \times 10^{-3}-8.8 \times 10^{-3}\right)\end{array}$ & $\begin{array}{c}8.6 \times 10^{-3} \\
\left(4 \times 10^{-3}-1.4 \times 10^{-2}\right)\end{array}$ & $\begin{array}{c}3.5 \times 10^{-3} \\
\left(1.5 \times 10^{-2}-6.1 \times 10^{-2}\right)\end{array}$ & $\begin{array}{c}5.8 \times 10^{-3} \\
\left(3.4 \times 10^{-3}-8.9 \times 10^{-3}\right)\end{array}$ & $\begin{array}{c}2.3 \times 10^{-3} \\
\left(1.3 \times 10^{-2}-3 \times 10^{-2}\right)\end{array}$ & $\begin{array}{c}6.8 \times 10^{-2} \\
\left(4.3 \times 10^{-2}-9.6 \times 10^{-2}\right)\end{array}$ \\
\hline $\begin{array}{l}\text { Cyclic } \\
\text { Alkanes }\end{array}$ & $\begin{array}{c}1.6 \times 10^{-4} \\
\left(0-4.5 \times 10^{-4}\right)\end{array}$ & $\begin{array}{c}6.1 \times 10^{-4} \\
\left(0-2 \times 10^{-4}\right)\end{array}$ & $0(0-0)$ & $\begin{array}{c}1.5 \times 10^{-3} \\
\left(4.4 \times 10^{-4}-3.2 \times 10^{-3}\right)\end{array}$ & $\begin{array}{c}4.3 \times 10^{-3} \\
\left(1.4 \times 10^{-3}-8.4 \times 10^{-3}\right)\end{array}$ & $\begin{array}{c}1.5 \times 10^{-4} \\
\left(0-3.5 \times 10^{-4}\right)\end{array}$ \\
\hline Alkenes & $\begin{array}{c}6.9 \times 10^{-4} \\
\left(1.9 \times 10^{-4}-1.3 \times 10^{-3}\right)\end{array}$ & $\begin{array}{c}1.1 \times 10^{-3} \\
\left(8.3 \times 10^{-5}-2.2 \times 10^{-3}\right)\end{array}$ & $\begin{array}{c}4.1 \times 10^{-3} \\
\left(4.7 \times 10^{-4}-9 \times 10^{-3}\right)\end{array}$ & $\begin{array}{c}1.3 \times 10^{-3} \\
\left(4.1 \times 10^{-4}-2.4 \times 10^{-3}\right)\end{array}$ & $\begin{array}{c}3.5 \times 10^{-3} \\
\left(1.2 \times 10^{-3}-6.3 \times 10^{-3}\right)\end{array}$ & $\begin{array}{c}9.8 \times 10^{-3} \\
\left(2.9 \times 10^{-3}-0.02\right)\end{array}$ \\
\hline Alkynes & (2) & $\begin{array}{c}1.2 \times 10^{-3} \\
\left(0-3.7 \times 10^{-3}\right)\end{array}$ & $\begin{array}{c}1.4 \times 10^{-3} \\
\left(1.4 \times 10^{-3}-3.8 \times 10^{-2}\right)\end{array}$ & $0(0-0)$ & $\begin{array}{c}1.3 \times 10^{-4} \\
\left(0-3.5 \times 10^{-4}\right)\end{array}$ & $\begin{array}{c}5.1 \times 10^{-4} \\
\left(0-2 \times 10^{-3}\right)\end{array}$ \\
\hline Alcohols & $\begin{array}{c}5.1 \times 10^{-2} \\
\left(2.7 \times 10^{-2}-8.2 \times 10^{-2}\right)\end{array}$ & $\begin{array}{c}3.7 \times 10^{-2} \\
\left(0-7.6 \times 10^{-2}\right)\end{array}$ & $1(0.076-2.9)$ & $\begin{array}{c}2.4 \times 10^{-2} \\
\left(4.3 \times 10^{-3}-5.4 \times 10^{-2}\right)\end{array}$ & $\begin{array}{c}0.23 \\
(0-0.72)\end{array}$ & $0.78(0.06-2.5)$ \\
\hline Aldehydes & $\begin{array}{c}8 \times 10^{-2} \\
\left(6 \times 10^{-2}-0.1\right)\end{array}$ & $\begin{array}{c}0.23 \\
(0.12-0.35)\end{array}$ & $0.32(0.16-0.52)$ & $\begin{array}{c}6.1 \times 10^{-2} \\
\left(3.5 \times 10^{-2}-0.1\right)\end{array}$ & $0.36(0.2-0.52)$ & $0.3(0.13-0.54)$ \\
\hline Ketones & $0.18(0.12-0.26)$ & $\begin{array}{c}0.65 \\
(0.15-1.58) \\
\end{array}$ & $0.4(0.2-0.7)$ & $0.18(0.13-0.27)$ & $0.4(0.16-0.8)$ & $0.49(0.33-0.69)$ \\
\hline Methane & $0.05(0.02-0.08)$ & $\begin{array}{c}0.016 \\
(0.03-0.095) \\
\end{array}$ & $0.23(0.08-0.43)$ & $0.06(0.04-0.08)$ & $0.13(0.095-0.18)$ & $0.38(0.19-0.62)$ \\
\hline
\end{tabular}

Table S7: Uncertainty in US06 vis-à-vis running emissions at $75^{\circ} \mathrm{F}$.

\begin{tabular}{|l|c|c|c|c|}
\hline \multirow{2}{*}{ Species } & \multicolumn{2}{|c|}{ Running } & \multicolumn{2}{c|}{ US06 } \\
\cline { 2 - 5 } & Mean, 95 \% C.I & Uncertainty & Mean, 95 \% C.I & Uncertainty \\
\hline Aromatics & $0.7(0.4-1)$ & 2.5 & $3(2-6)$ & 3 \\
\hline Alkanes & $1.15(0.5-2.0)$ & 4.0 & $3.6(2.5-4.8)$ & 2.0 \\
\hline Cyclic Alkanes & $0(0-0)$ & $\mathrm{n} / \mathrm{a}$ & $0.25(0.08-0.4)$ & 5.0 \\
\hline Alkenes & $0.1(0.13-0.3)$ & 16 & $2.2(0.97-3.56)$ & 3.7 \\
\hline Alkynes & $0.03(0.003-0.06)$ & 20 & $0.08(0-0.19)$ & infinity \\
\hline Alcohols & $7.7(0.7-18)$ & 26 & $2.1(0.7-3.7)$ & 5.3 \\
\hline Aldehydes & $2.2(1.4-2.9)$ & 2 & $0.76(0.3-1.5)$ & 5 \\
\hline Ketones & $0.4(0.2-0.7)$ & 3.5 & $0.16(0.07-0.25)$ & 3.6 \\
\hline Methane & $2.7(1-5)$ & 5 & $5.1(3.5-6.5)$ & 1.9 \\
\hline
\end{tabular}

Table S8: Probability of speciation fractions being equal across all temperatures for the US06 cycle.

\begin{tabular}{|l|c|c|c|}
\hline & \multicolumn{3}{|c|}{ US06 } \\
\cline { 2 - 4 } & $\mathbf{0}^{\mathbf{0}} \mathbf{F} / \mathbf{7 5}^{\mathbf{0}} \mathbf{F}$ & $\mathbf{2 0}^{\mathbf{0}} \mathbf{F} / \mathbf{7 5}^{\mathbf{0}} \mathbf{F}$ & $\mathbf{0}^{\mathbf{0}} \mathbf{F} / \mathbf{2 0} \mathbf{\mathbf { }} \mathbf{F}$ \\
\hline Aromatics & $<9 \times 10^{-4}$ & $<9 \times 10^{-4}$ & $<9 \times 10^{-4}$ \\
\hline Alkanes & $<10^{-4}$ & $<9 \times 10^{-4}$ & $<10^{-4}$ \\
\hline Cyclic Alkanes & $<10^{-4}$ & $<10^{-4}$ & $<10^{-4}$ \\
\hline Alkenes & $<10^{-4}$ & $0.15^{-4}$ & $<10^{-4}$ \\
\hline Alkynes & $<10^{-4}$ & $<9 \times 10^{-4}$ & $<10^{-4}$ \\
\hline Alcohols & $<10^{-4}$ & $<9 \times 10^{-4}$ & $<10^{-4}$ \\
\hline Aldehydes & $<10^{-4}$ & $<10^{-4}$ & $<10^{-4}$ \\
\hline Ketones & $<9 \times 10^{-4}$ & $<10^{-4}$ & $<9 \times 10^{-4}$ \\
\hline Methane & $<10^{-4}$ & $<10^{-4}$ & $<10^{-4}$ \\
\hline
\end{tabular}




\section{Unspeciated VOCs}

An important issue in speciation is the fraction of unspeciated VOCs. Jathar et al. (2014) indicated that TOG emissions from combustion sources include a significant amount of unspeciated organic mass, which could significantly influence the formation of secondary organic aerosol. A commonly used technique of quantifying the unspeciated mass is to compare the measurements from two different methods (e.g. Jathar et al., 2014). The authors compared the non-methane hydrocarbon totals measured by the Flame Ionization Detector (FID) to the speciated summation from the Gas-Chromatography-Flame Ionization Detector (GC-FID). Their study revealed that the FID totals were higher than the GC-FID, so the difference (FID minus GC-FID) could quantify the unspeciated compounds. We used their approach; a sample result from the US06 driving cycle is plotted in Figure S4. Similar trends were reported by the other cycles and temperatures. The GC vs. GC-FID show trends which are opposite to those observed by Jathar et al. (2014), i.e. the FID totals bias lower than the GC-FID (FID = 0.6*GC-FID; $\mathrm{R}=0.83)$. The results demonstrate the difficulty in quantifying unspeciated compounds. There are several reasons why the trends in this study are opposite that of Jathar et al. (2014). For example, the FID measurements, were made in the test cell laboratory, under different sampling conditions than the GC-FID at Allen Park. Additionally, the FID and GC-FID are calibrated using different compounds (propane vs. individual hydrocarbon species).

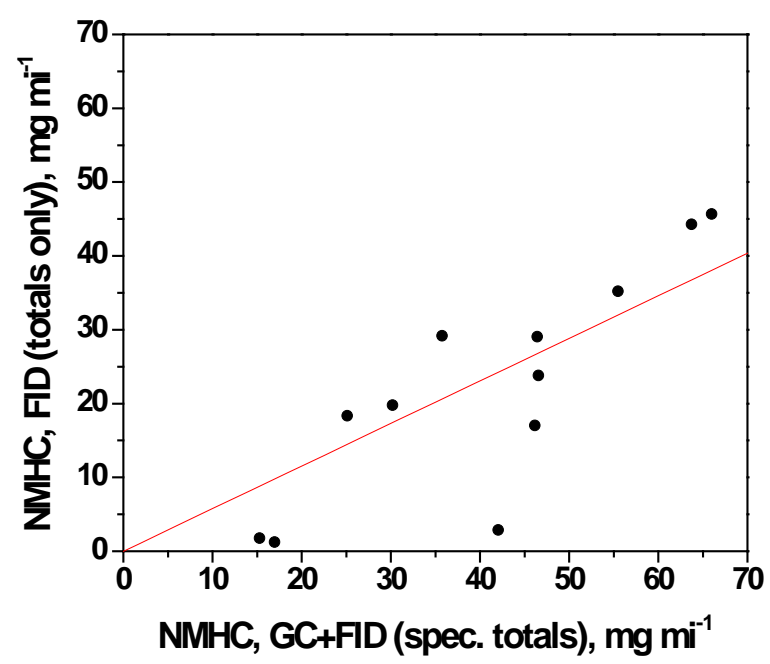

Figure S4: Comparison of NonMethane Hydrocarbon emission factors using two different methods to quantify unspeciated compounds. Data are plotted for the US06 cycle, the FTP75 cold start and composite emissions showed similar trends. 
NMOG-to-NMHC ratios

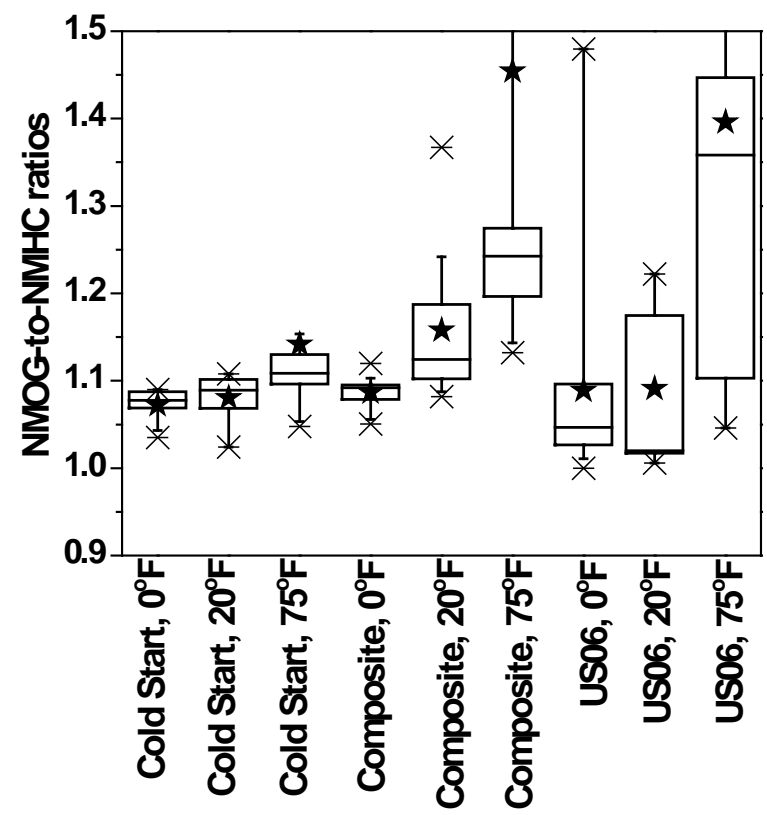

Figure S5: Box plot of NMOG-NMHC ratios for the vehicle fleet tested. Star represents the fleet-average. The calculation was done as follows:

$$
\begin{gathered}
N M O G=N M H C \sum_{i=1}^{4} \text { SpeciationConstant }+ \text { oxySpeciation } \times \text { voltoWeightPercentO }^{2} y_{i} \\
\times \text { oxyVolumei }
\end{gathered}
$$

$i$ = one of four gasoline oxygenates: ethanol, methyl tert-butyl ether (MTBE), ethyl tert-butyl ether (ETBE), or tert-amyl methyl ether (TAME). Only ethanol was considered because the others were not present in the fuel speciation.

SpeciationConstant $=$ NMOG/NMHC conversion factor when the gasoline has no oxygenate volume, taken to be 1.0149 .

volToWtPercentOxyi = term used to convert from the oxygenate percentage by volume (vol\%) to the mass percentage of oxygen in the fuel(mass\%). This was taken to be 0.37 for ethanol.

oxyVolumei $=$ the percent volume of each gasoline oxygenate in the respective fuel. This was taken to be $10 \%$ as per the fuel speciation.

\section{References}

Jathar, S.H., Gordon, T.D., Hennigan, C.J., Pye, H.O.T., Pouliot, G., Adams, P.J., Donahue, N.M., Robinson, A.L. Unspeciated organic emissions from combustion sources and their influence on the secondary organic aerosol budget in the United States. Proc. Natl. Acad. Sci. U.S.A, 2014, 111(29), 10473-10478. 